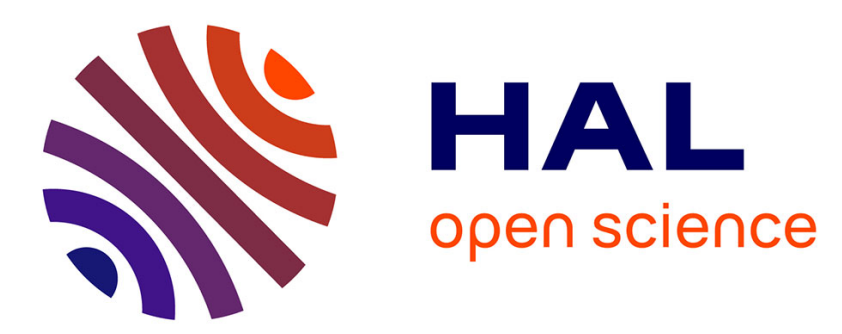

\title{
A hybrid approach for the evaluation of rail monitoring and maintenance strategies for the Grand Paris Express new metro
}

Laurent Bouillaut, Olivier Francois, Yves Putallaz, Clément Granier, Christophe Cieux

\section{To cite this version:}

Laurent Bouillaut, Olivier Francois, Yves Putallaz, Clément Granier, Christophe Cieux. A hybrid approach for the evaluation of rail monitoring and maintenance strategies for the Grand Paris Express new metro. International Journal of Performability Engineering, 2020, 16, pp 1685-1697. 10.23940/ijpe.20.11.p1.16851697 . hal-03079167

\section{HAL Id: hal-03079167 https://hal.science/hal-03079167}

Submitted on 17 Dec 2020

HAL is a multi-disciplinary open access archive for the deposit and dissemination of scientific research documents, whether they are published or not. The documents may come from teaching and research institutions in France or abroad, or from public or private research centers.
L'archive ouverte pluridisciplinaire HAL, est destinée au dépôt et à la diffusion de documents scientifiques de niveau recherche, publiés ou non, émanant des établissements d'enseignement et de recherche français ou étrangers, des laboratoires publics ou privés. 


\title{
A hybrid approach for the evaluation of rail monitoring and maintenance strategies for the Grand Paris Express new metro
}

\author{
Laurent Bouillaut*a, Olivier François ${ }^{\mathrm{a}}$, Yves Putallaz ${ }^{\mathrm{b}}$, Clément Granier ${ }^{\mathrm{b}}$, Christophe Cieux ${ }^{\mathrm{c}}$ \\ ${ }^{a}$ Université Gustave Eiffel, COSYS/GRETTIA, 77447 Marne-la-Vallée, France \\ ${ }^{b} I M D M$ - Infra Consulting, 1800 Vevey, Switzerland \\ ${ }^{c}$ Société du Grand Paris, Systems and Safety unit, 93200 St Denis, France \\ *E-mail address: laurent.bouillaut@univ-eiffel.fr
}

\begin{abstract}
Three years after its first 2010 enactment, the French government proposed a timeline for the development of a new metro network providing new rapid transit lines in the Ile de France region. Implemented by the Société du Grand Paris (SGP), the Grand Paris Express (GPE) thus became the largest transport project in Europe. As in any new railway project, a safety record must be established by the SGP. Among all criteria that had to be investigated, the ability of the network manager to prevent and detect broken rails was a particularly sensitive point. Indeed, beyond the obvious consequences induced by a broken rail impacting the safety of passengers, such an event has a very strong impact on the availability of the infrastructure, which is a key point for all metro line automation projects. The SGP therefore required some decision support tools to enable us to evaluate the consequences of a broken rail on the network operating conditions and, moreover, to determine the best monitoring strategy and the adequacy of the maintenance policy to prevent broken rails.

Based on a former study commissioned by RATP (the historic operator and infrastructure manager of the Paris metro network) dealing with the evaluation and optimization of detection and prevention of broken rails in a metro line automation context, the SGP wanted to possess an equivalent decision support tool customised to the needs and the characteristics of the GPE. Nevertheless, in the GPE context, the network that was considered does not yet exist. Moreover, no equivalent system exists in the world. No feedback databases are therefore available to estimate reliability parameters necessary for such decision support tools. Therefore, the present paper proposes an original approach combining physical modelling of rail deterioration with statistics to overcome this limitation.
\end{abstract}

Keywords: Reliability analysis; maintenance optimization; broken rails; stochastic modelling; mechanical modelling of rail degradation; Bayesian networks; hybrid learning approach;

(C) 2019 Totem Publisher, Inc. All rights reserved.

\section{Introduction}

Three years after its first 2010 enactment, the French government proposed a timeline for the development of a new metro network providing new rapid transit lines in the Ile de France region. Implemented by the Société du Grand Paris (SGP), the Grand Paris Express (GPE) thus became the largest transport project in Europe. The GPE consists of a fundamental redesign and focus on the public transport network of the whole metropolitan area around Paris, among which a ring route around Paris (line 15, named the "red line") is being added.

As in any new railway project, a safety record must be established by the SGP. Among all criteria that had to be investigated, the ability of the network manager to prevent and detect broken rails was a particularly sensitive point. Indeed, beyond the obvious consequences induced by a broken rail that can impact the safety of passengers (such as derailment or rail gauge commitment), such failures of the railway infrastructure have also very strong systematic impacts on the quality of service (in terms of availability, regularity, etc.).

The SGP therefore required some decision support tools to enable us to evaluate the consequences of a broken rail on the network operating conditions and, moreover, to determine the best monitoring strategy and an adequate maintenance policy to prevent broken rails.

Considering the earlier "StatAvaries" study, commissioned by RATP (the historic operator and infrastructure manager of the Paris metro network), dealing with the evaluation and optimisation of detection and prevention of broken rails in a metro

* Corresponding author.

E-mail address: author@institute.xxx . 
line automation context, the SGP wanted to possess an equivalent decision support tool customised to the needs and the characteristics of the GPE.

As the developer of the StatAvaries tool [1], we were therefore invited by the SGP to conduct this new study named VirMaLab - Red line. Based on the VirMaLab (Virtual Maintenance Laboratory) generic approach [2], the StatAvaries tool has a modular structure for modelling the rail degradation processes (considering some contextual variables such as the position of the rail, its stiffness, the operating characteristics, etc.), the supervision strategy (considering the monitoring device properties such as good detection, false alarms and confusion rates, periodicity, detection framework, etc.), and the maintenance policy (with a range of maintenance actions from punctual repairs to preventive maintenance actions but also imperfect maintenance). The VirMaLab approach uses the Dynamic Bayesian Network (DBN) formalism [3] and, more specifically, a specific DBN structure, named the Graphical Duration Model (GDM) [4], which allows the Markovian assumption induced by the standard DBN to be overcome. Indeed, considering constant transition rates is particularly inappropriate in the railway field.

For all stochastic approaches to reliability analysis, one of the key points of such modelling is the parameter learning process. In a VirMaLab tool context, learning is commonly performed using a feedback database. In many studies dealing with very efficient learning algorithms, the main point is to have a deep enough feedback database to avoid the problems of over- or underrepresentation induced by small data sets [5]. In the railway field, such return of experience is generally available. Indeed, the dynamic behaviour of train axles, the variability of support conditions and the presence of residual stresses from thermal treatments during the manufacturing process and welding all contribute to the complexity of rail mechanical behaviour. Accurately predicting the evolution of rail conditions based solely on physical models would thus require the processing of tremendous amounts of data regarding train movements and infrastructure conditions, much of this information being generally unavailable. Therefore, a stochastic approach is generally considered.

Nevertheless, in the GPE context, this network does not yet exist. Moreover, no one equivalent system exists in the world. The VirMaLab - Red line project therefore had to consider a new approach for learning the parameters for its maintenance model. In the StatAvaries study, the rail deterioration module used experience records to bypass the uncertainty in the rail mechanical behaviour. Particularly, the Weibull distribution describing the different stages of the rail deterioration process has been obtained through machine learning on a set of measurements of the rail condition of the existing Line 7 of the Parisian Subway. However, such records cannot exist for an infrastructure that is still in the design phase, such as line 15 .

The present paper therefore proposes an original approach combining physical modelling of rail deterioration with statistics to overcome this limitation.

After a first section dedicated to the generic approach considered for developing decision support tools for the evaluation of maintenance strategies and its scientific formalism, the industrial context of this study will be described in detail. Then, the "mecanico-stochastic" learning process will be described in detail in section 3, and some results will be introduced and discussed in section 4 before some conclusions and prospects.

\section{VirMaLab - a decision support tool for maintenance policy evaluation and optimisation}

\subsection{Generalities and main properties of the approach}

The aim of our work is to propose models able to settle, for a predetermined context and for operating constraints, the optimal diagnosis parameters and the most adapted maintenance policy. The generic approach used for building a VirMaLab decision support tool for determining optimal maintenance strategies is divided into three modules:

- The first module consists of the mathematical modelling of the physical state of the system and the time evolution of its various components. This strategy means being able to determine if the system is still fault-free after a given operating time, knowing its initial state and the operating parameters. In contrast, if a defect appears, it is necessary to determine, from a predefined list of possible defects, the state of damage to the system. Due to the variability of contexts and the system size, a probabilistic approach is adopted. In this way, the probability of facing a given state is estimated rather than its deterministic existence. One of the most commonly used approaches is based on Markov chains, frequently modelled by DBN. A Markov chain is a discrete sequence of states, taking their value in a discrete state space, that follows the Markov property (knowing the present, the past does not have any impact on what will happen in the future). Therefore, dwell times in each state must necessarily be exponentially distributed. If some systems confirm this assumption, most industrial applications exhibit nonMarkovian behaviours. In this case, Markovian degradation process modelling can introduce non-negligible biases. Then, the estimated maintenance parameters can be far from optimal results [6]. To overcome this drawback, the VirMaLab approach introduces semi-Markovian modelling of degradation processes that can fit all kinds of dwell time distributions: graphical duration models (GDMs) [4]. 
- The second module consists of modelling diagnostic devices and their setting parameters. According to supervision results, each reference frame recommends the use of a maintenance action adapted to the current estimated state of the system. When a maintenance action is performed, the state of the system and its degradation process must be updated to consider the impact of the maintenance action.

- Finally, the last phase consists of quantifying the maintenance policy in terms of safety, costs, availability, regularity, etc.

Then, with such a tool, one can evaluate various maintenance strategies and determine, for a given cost function, the best set of maintenance and monitoring parameters. The learning of such modelling can be done with both expert advice and feedback databases.

\subsection{Dynamic Bayesian Networks: main properties}

Considering discrete and finite state space systems, all the modelling is based on the DBN formalism. Since the aim of this paper is not to detail once again the VirMaLab approach nor to justify the use of one formalism rather than another, the DBN will be very briefly introduced in this subsection. For more details on these points, the reader may refer to [2], [3] and [4]. Formally, a Bayesian network $(\mathrm{BN})$, denoted by $\mathcal{M}$ is defined as a pair $\left(\mathcal{G},\left\{p_{n}\right\}_{1 \leq n \leq N}\right)$ where:

- $\mathcal{G}=(X, \varepsilon)$ is a directed acyclic graph giving a qualitative description of the BN. The graph nodes and the associated random variables are both represented by $X=\left\{X_{1}, \ldots, X_{N}\right\}$, with values in $\mathcal{X}=\mathcal{X}_{1} \mathrm{x} \ldots \mathrm{x} \mathcal{X}_{N}$. $\varepsilon$ is the set of edges encoding the conditional independence relationships among these variables.

- $\left\{p_{n}\right\}_{1 \leq n \leq N}$ is a set of conditional probability distributions (CPDs) associated with the random variables. These distributions aim to quantify the local stochastic behaviour of each variable.

In addition, both qualitative (i.e., $\mathcal{G}$ ) and quantitative (i.e., $\left\{p_{n}\right\}$ ) parts of $\mathcal{M}$ can be learned automatically if some complete or incomplete data or experts' opinions are available [7]. Using BN is also particularly interesting because of the ease of knowledge propagation through the network. Indeed, various inference algorithms allow computing the marginal distribution of any subset of variables.

From a dynamic behaviour modelling point of view, the time extension of BN provides a convenient formalism to represent discrete sequential systems. Indeed, DBNs are dedicated to modelling data that are sequentially generated by some complex mechanisms (time-series data, biosequences, number of mechanical solicitations before failure, etc.). Therefore, DBNs are frequently used to model Markov chains. Formally, a DBN is defined by a pair of BNs $\left(\mathcal{M}_{\text {ini }}, \mathcal{M}_{\rightarrow}\right)$ where:

- $\mathcal{M}_{i n i}=\left(\mathcal{G}^{i n i},\left\{p_{n}^{i n i}\right\}_{1 \leq n \leq N}\right)$ is a BN modelling the initial distribution of $X$, denoted $p_{i n i}$.

- $\mathcal{M}^{\rightarrow}=\left(\mathcal{G}^{\rightarrow},\left\{p_{t, n}\right\}_{2 \leq t \leq T ; 1 \leq n \leq N}\right)$ defines the transition model of the considered process, which represents the distribution of $X_{t}$ knowing $X_{t-1}$, denoted $p$, etc.

Figure 1(a) introduces a DBN modelling the Markov chain of the sequence $X=\left(X_{1}, \ldots, X_{\mathrm{N}}\right)$ taking its values in the set $\mathcal{X}$. This DBN is described by the $\operatorname{pair}\left(\mathcal{M}_{i n i}, \mathcal{M}^{\rightarrow}\right)=\left(\left(X_{1}, p_{1}\right),\left(\mathcal{G}^{\rightarrow}, Q^{s y s}\right)\right)$ where $\mathcal{Q}^{\text {sys }}$ denotes the transition matrix of a Markov Chain, quantifying the probability of $X_{t}$ knowing $X_{t-1}$.

If this approach is perfectly adapted to model the dynamics of systems, it induces a strong assumption on the sojourn time distribution in each state of the system. Indeed, as in all Markovian approaches, transition rates are assumed to be constant and, therefore, sojourn times are necessarily geometrically distributed. In many industrial applications, such an assumption can introduce strong bias in degradation modelling that cannot be foreseen in the context of reliability based optimisation of maintenance. To overcome this drawback, a specific DBN, designated the graphical duration model, was proposed and will be introduced briefly in the next paragraphs.

The graphical duration model is a specific DBN that uses a semi-Markov approach. The main idea is to deal with the couple $\left(X_{t}, S_{t}\right)$ rather than the single variable $\left\{X_{t}\right\}$, where $S_{t}$ denotes the time remaining in the current state denoted by $X_{t}$. Figure 1(b) introduces the structure of a DBN modelling a GDM. The solid lines define the basic structure of the GDM; dashed lines indicate optional items (detailed in [4]), and red bold edges characterise dependences between time slices.

A GDM is therefore defined by the pair $\left(\mathcal{M}_{\text {ini }}, \mathcal{M}^{\rightarrow}\right)$ with:

- $\quad \mathcal{M}_{i n i}=\left(\mathcal{G}^{\text {ini }},\left(\alpha_{1}, F_{1}\right)\right)$ where $\alpha_{1}$ and $F_{1}$ denote the initial distributions of $X_{1}$ and $S_{1}$, respectively.

- $\mathcal{M}^{\rightarrow}=\left(\mathcal{G}^{\rightarrow},\left(Q^{\rightarrow}, F^{\rightarrow}\right)\right)$ is characterised by two transition distributions: $Q^{\rightarrow}$ is the natural state change distribution, and $F^{\hookrightarrow}$ is the dwell time distribution, both described by equations (1) and (2) and widely detailed in [4] [6]. 


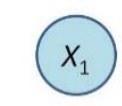

Initial model

$\mathcal{M}_{\text {ini }}$

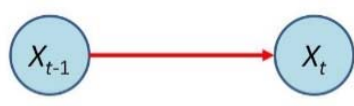

Transition model

$\mathcal{M} \rightarrow$

(a)

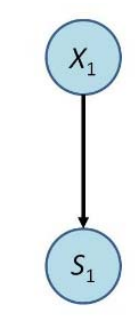

Initial model $\mathcal{M}_{\text {ini }}$

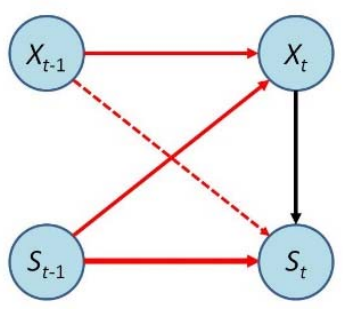

Transition model $\mathcal{M} \rightarrow$

(b)

Figure 1. DBN modelling dynamic behaviour of random variables: (a) Markovian standard approach (b) Semi-Markovian MGD.

$$
\begin{aligned}
& F^{\rightarrow}=p\left(S_{t}=s \mid X_{t}=x, S_{t-1}=s^{\prime}\right)
\end{aligned}
$$

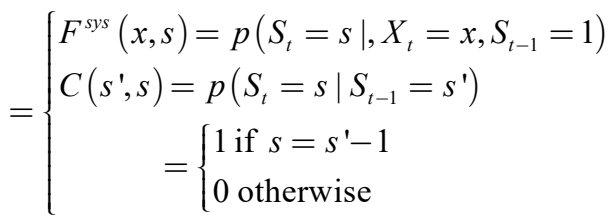

$$
\begin{aligned}
& Q^{\rightarrow}=p\left(X_{t}=x \mid X_{t-1}=x^{\prime}, S_{t-1}=s^{\prime}\right) \\
& =\left\{\begin{array}{c}
Q^{s y s}\left(x^{\prime}, x\right)=p\left(X_{t}=x \mid, X_{t-1}=x^{\prime}, S_{t-1}=1\right) \\
I\left(x^{\prime}, x\right)=p\left(X_{t}=x \mid X_{t-1}=x^{\prime}, S_{t-1}>1\right)
\end{array}\right.
\end{aligned}
$$

In addition, the structure of a GDM, introduced in Figure 1(b), shows that processes $\left(X_{t}\right)$ and $\left(S_{t}\right)$ are not Markovian since $X_{t-1} \not \Perp X X_{t+1} \mid X_{t}$ and $S_{t-1} \not \Perp S_{t+1} \mid S_{t}$. where the notation $A \not \Perp B \mid C$ means that variables A and B are statistically dependent given $\mathrm{C}$. However, the GDM structure leads to:

$$
\left(X_{t-1}, S_{t-1}\right) \Perp\left(X_{t+1}, S_{t+1}\right) \mid\left(X_{t}, S_{t}\right)
$$

Therefore, the set $\left(X_{t}, S_{t}\right)$ engendered by a GDM is Markovian, despite $\left(X_{t}\right)$ not being Markovian.

From a practical point of view, this approach allows specifying arbitrary state dwell time distributions by contrast with a classic Markovian framework in which all dwell times must be exponentially distributed. This modelling is therefore particularly interesting as soon as the question is to capture the behaviour of a given system subjected to a particular context and a complex degradation distribution. More details on this GDM (quantitative description, optional context description, etc.) can be found in [4]. Finally, various costs can be considered using utility nodes, introduced in BN theory to provide decision support tools named influence diagrams [8].

\section{A "mecanico-stochastic" learning process for the evaluation of rail degradation dynamics}

\subsection{Introduction of the industrial context of the study}

In a former study commissioned by the Paris public transport network operator and infrastructure manager (RATP) ten years ago, the StatAvaries project aimed to propose a maintenance model for the evaluation of the ability of the RATP to detect and prevent broken rails in the context of the steel-wheel metro line automation. An original VirMaLab modelling of a maintenance strategy was therefore proposed. Finally, the multimodel extension introduced for this study considered multiple temporal sampling events, satisfying both the degradation dynamic and the accuracy required to correctly quantify the impact of broken rails and their related false alarms to support RATP in its decisions. All details of this study can be found in [1].

Due to the new GPE project, SGP found it necessary to address the difficult issue of the detection and prevention of broken rails in a steel-wheel automated train context. Indeed, the dynamic behaviour of train axles, the variability of support conditions and the presence of residual stresses from thermal treatments during the manufacturing process and welding all contribute to the complexity of rail mechanical behaviour [9].

The rail deterioration module of StatAvaries used experience records to bypass the uncertainty associated with the rail mechanical behaviour. In particular, Weibull distributions describing the different stages of the rail deterioration process were 
learned from the REX database of the Parisian subway line 7 [1]. Obviously, such records cannot yet exist for the line 15 infrastructure. The present paper proposes an approach combining physical modelling of rail deterioration with statistics to overcome this limitation.

To simplify the analysis and according to the International Union of Railways standards, the rail states along the main deterioration process were clustered into four classes: Ok (the rail has no defect), $\mathrm{X}_{1}$ (internal cracks $<2.4 \mathrm{~mm}$ ), $\mathrm{X}_{2}$ (internal cracks $<30 \mathrm{~mm}$ ) and BR (broken rail).

A metro line is composed of hundreds of elementary rail sections (generally between 5 and 18 metres long), with various ages, various states, etc.

For reasons of complexity, the development of a degradation model of a complete line is, therefore, unrealistic. To tackle this issue, a given line (or portion of a line) was therefore assumed to be the sequence of a set of independent elementary rail sections (as illustrated in Figure 2), 18 metres long.

The proposed model focusses on one of these elementary sections. Then, the results are extrapolated to larger line sections to obtain reliability indicators on the considered portion of the line.

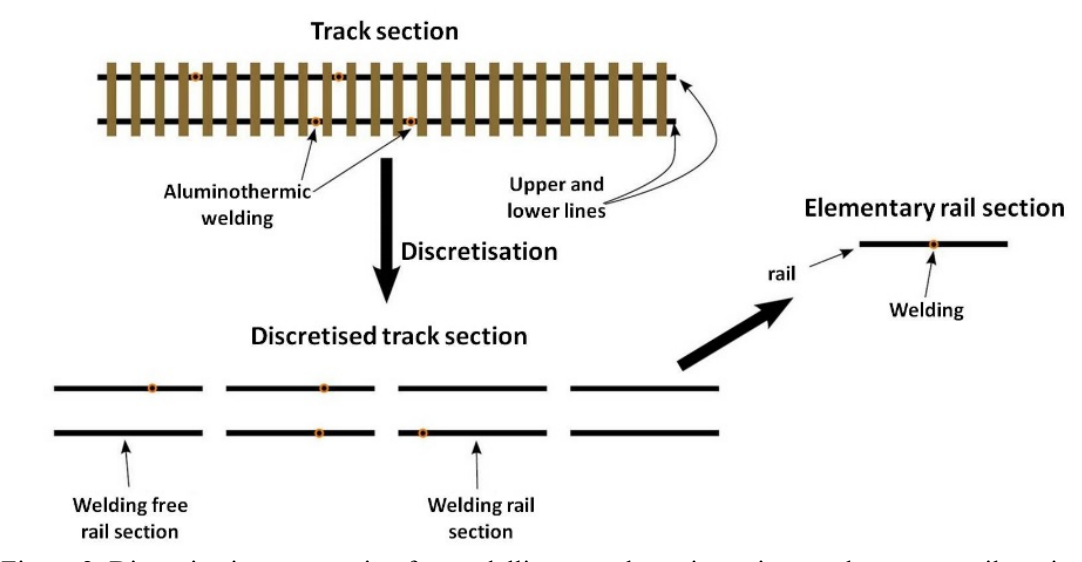

Figure 2: Discretization assumption for modelling a track section using an elementary rail section

\subsection{General methodology}

Adapting the StatA varies rail deterioration model to line 15 means adjusting the Weibull distributions describing the dwell time of a rail in the four deterioration states considered in this new context. Herein, a method by comparison is proposed to extend the experience acquired on line 7 to line 15 by using a physical model able to quantify the relative influence of physical parameters on rail conditions.

The physical model provides an estimation of the stress level in the rails depending on their cross-section, the horizontal and vertical alignment of the track, the characteristics of the rolling stock and operations (volume of traffic, speed). Based on the principles of fracture mechanics [10], the dwell times of flaws in each deterioration state can then be computed.

The rail deterioration model is used to calculate the evolution of a series of common flaws [11], successively subjected to the contexts of line 7 and line 15. In other words, the dwell times of several generic flaws are computed for every homogeneous track segment in terms of the type of rails, annual load level, track alignment and allowable speed.

Based on the length of the homogeneous segments, the weighted average of the dwell times is computed for each line and each deterioration state. The values obtained are then used to adjust the expected values of the Weibull distributions to the context of line 15 as follows:

$$
\mu_{X, L 15}=\frac{t_{X, L 15}}{t_{X, L 7}} \cdot \mu_{X, L 7}
$$

where:

- $\mu_{X, L i}$ defines the expected value of the Weibull distribution for the dwell time of a rail in state $X$, on line $i$.

- $t_{X, L i}$ is the average dwell time of an "average flaw" on line $i$ as calculated by the rail deterioration model.

The relative shape of the dwell time distribution around the expected value is assumed to be equivalent for both lines. As a consequence, the shape parameter of the Weibull distributions should remain constant. The scale parameter, however, is a function of the expected value and must be adjusted to the context of line 15 [12].

$$
\alpha_{X, L_{15}}=\frac{\mu_{X, L_{15}}}{\Gamma\left(1+\frac{1}{\beta_{X, L_{15}}}\right)}
$$


where $\mu_{X, L 15}, \alpha_{X, L 15}$ and $\beta_{X, L 15}$ are the expected value, the scale and the shape parameters of the Weibull distribution describing the dwell time of a rail in the deterioration state $\mathrm{X}$, respectively, on line 15.

\subsection{Physical modelling of rail degradation}

The model is based on the principles of structural mechanics and fracture mechanics. Structural mechanics concentrates on analysing the behaviour of structural members subjected to loads, particularly their internal stresses and strains. Fracture mechanics [10] is concerned with the propagation of cracks inside structural members subjected to load cycles. The concept of load cycle can be understood as the complete process of loading and unloading a structural member. The concept of load cycles can be understood as the complete process of loading and unloading a structural member. Any increase in the tensile or shear stresses on the edge of a crack participates in its propagation. In the railway field, the moving train axles can be seen as cyclic loads stressing the rails. A schematic representation of the effect of a moving axle load on a flaw located in the head of a rail is provided in Figure 3 [13]. The development of rail defects under the influence of cyclic loading is composed of three phases:

- the nucleation phase [14]: initiation of microcracks induced by the plastic movement of dislocations within the crystalline structure of the metal,

- the propagation phase: controlled propagation of microcracks until a critical size is reached,

- the failure phase: fast and uncontrolled propagation of cracks leading to the failure of the cross-section.
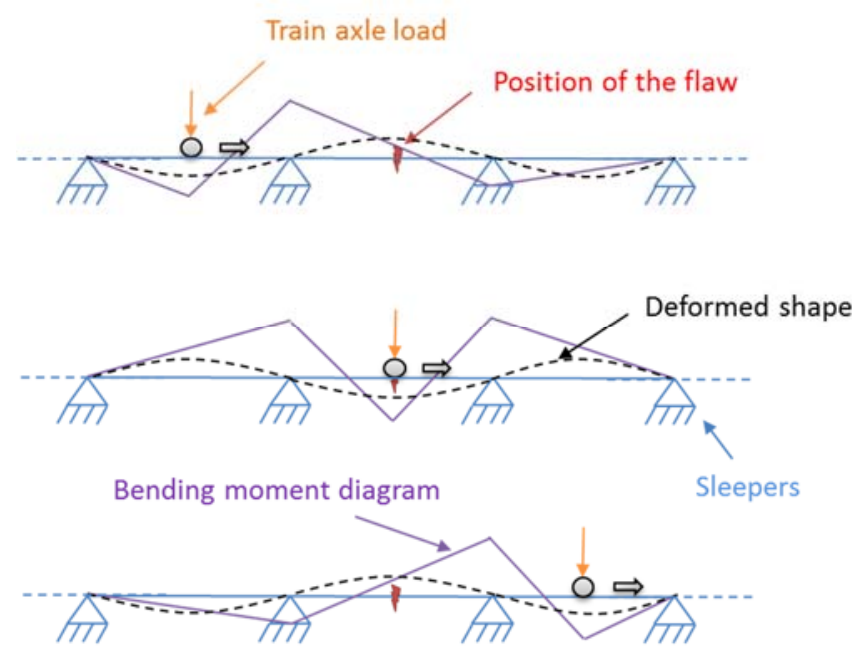

Cycle n-1: Tensile stresses due to bending

Cycle n: Compressive stresses due to bending + Hertz contact stresses

Cycle $n+1$ : Tensile stresses due to bending

Figure 3: Cyclic loading of a flaw located in the head of a rail by a moving train axle

The nucleation phase depends mainly on dislocations initially present in the material and then on the manufacturing process. We assume here that the rails of both line 7 and line 15 comply with the same quality standards. Following this hypothesis, the probability of new flaws emerging from dislocations is the same on both lines. Therefore, the nucleation phase does not cause the expected value of the Weibull distributions to vary from one line to another.

Furthermore, the failure phase is generally very short. The influence of the failure phase on the dwell time of a flaw in state $\mathrm{X}_{2}$ is thus negligible. Consequently, the physical model presented herein concentrates on the propagation phase, where the average duration is likely to change from line 7 to line 15 . The physical model allows calculating the stress cycles inside the rails generated by the passing of trains, the propagation velocity of the cracks and then the corresponding dwell times in the states $\mathrm{Ok}, \mathrm{X}_{1}$ and $\mathrm{X}_{2}$. The 4 stresses considered by the model are:

- the Hertz contact stresses [15]:

$$
\sigma_{H e r t z}=\sqrt{\frac{\pi \cdot E}{64 \cdot\left(1-v^{2}\right)} \cdot \frac{Q}{R \cdot b}}
$$

where $E$ is the modulus of elasticity of rail steel,

$v$ : Poisson's ratio of rail steel, on average equal to 0.3 ,

$Q$ : the load exerted by a train axle on the rail,

$R$ : the radius of the train wheel,

$b$ : half of the width of the contact surface between the rail and the wheel, on average equal to $6 \mathrm{~mm}$. 
For an adhesion coefficient of 0.3 , the maximum shear stress is approximately 0.3 times the peak Hertzian contact stress [16]. In average this value is reached at a depth of $5 \mathrm{~mm}$ under the surface of the rail [16].

The lateral position of the wheel on the rolling surface tends to vary due to the geometry of the track-axle system. Given that it would be almost impossible to calculate the level of stresses in every possible configuration and that the lateral positioning of the analysed defects is fully theoretical and could vary in real applications, it has been chosen to neglect this parameter in the mode

- the vertical (7) and horizontal (8) bending stresses:

with:

$$
\begin{aligned}
& \sigma_{b, v}=\frac{M_{z}}{I_{z}} \cdot z \\
& \sigma_{b, h}=\frac{M_{y}}{I_{y}} \cdot y
\end{aligned}
$$

$M_{z}$ and $M_{y}$, respectively, the vertical and horizontal bending moment,

$I_{z}$ and $I_{y}$, respectively, the second moment of area of the rail cross-section about the vertical or horizontal axis, $z$ and $y$, respectively, the vertical and horizontal distances between the bending neutral axis and the point of interest.

- the residual stresses due to laminations and welding [17, 18].

Thermal stresses are neglected for two reasons: railways are mainly underground. Therefore, temperature does not vary much, and the average effect of temperature fluctuations over a year should be close to zero given that rails are welded at the average annual temperature. Finally, the stresses resulting from the bending of the rail head on the web are also neglected since they are very small.

Rails are modelled as continuous beams supported by the sleepers. These supports are considered stiff. This simplifying assumption leads to a slightly pessimistic estimation of the bending stresses in the case of a ballasted track (line 7). However, the influence of bending stresses on the development of flaws located in the head of rails is relatively small in comparison to the influence of contact stresses.

The propagation speed of a flaw depends on the difference in the stress intensity factor defined by (9) [10].

$$
\Delta K=Y \cdot K_{t} \cdot \Delta \sigma \cdot \sqrt{\pi \cdot a}
$$

with:

$Y=Y_{e} \cdot Y_{f a} \cdot Y_{s}$ is the correction factor depending on the shape and the position of the crack and on the dimensions of the cross-section of rail

$K_{t}$, the factor of stress concentration,

$\Delta \sigma$, the tensile stress difference induced by a load cycle at the front of the crack,

$a$, the size of the crack.

Paris' law (10) relates the propagation speed of a crack to the difference in the stress intensity factor.

$$
\frac{d a}{d N}=C \cdot(\Delta K)^{m}
$$

where $N$ is the number of load cycles, $C=1.1 \times 10^{-11}$ and $m=3$ (for steel).

The axle loads are computed from:

- the gross weight of the trains,

- an estimation of the number of passengers.

In the case of GPE line 15, forecasts of passenger flows are used to estimate the axle load during peak hours. During the slack period, a load of 0.5 passengers $/ \mathrm{m}^{2}$ is considered. However, no data on the passenger flows were available for line 7 of the Paris suburban area. Therefore, a load of 4 passengers $/ \mathrm{m}^{2}$ during the peak hours and of 0.5 passengers $/ \mathrm{m}^{2}$ during the slack period was considered. A sensitivity analysis will be carried out to assess the influence of this assumption on the Weibull distributions. Static loads are multiplied by a dynamic amplification factor defined by (7) [19].

$$
\gamma_{d y n}=1+0.14 \cdot \frac{v[\mathrm{~km} / \mathrm{h}]}{120}
$$


where $\mathrm{v}$ is the speed of the train.

For every homogeneous track segment, the dwell times of a series of common flaws in the deterioration states $O K, X_{1}$ and $X_{2}$ are computed. The dwell times of what is referred to as an "average flaw" are obtained by averaging the dwell times of all the flaws that were considered in the corresponding deterioration state. These common flaws are listed below:

- in tangents: internal cracks at mid-span between two sleepers, internal cracks above a sleeper, inclusion close to the rolling surface above a sleeper (weld) and inclusion in the gauge corner of the rail's head above a sleeper (weld)

- in curves: internal cracks on the field side of the head of the high rail at mid-span between two sleepers, internal cracks on the gauge side of the low rail above a sleeper, inclusion in the field-side corner of the high rail's head (weld) and inclusion in the gauge side corner of the low rail.

The size of the initial cracks is assumed to be $0.1 \mathrm{~mm}$ in the case of inner cracks and $0.4 \mathrm{~mm}$ in the case of welding defects while Inner cracks are also considered to initiate $12.5 \mathrm{~mm}$ under the rolling surface [20].

\section{Some illustrative results}

\subsection{Dwell time generalities}

Figure 4 shows the propagation curve of a welding defect located on a segment of line 7 . Table 1 shows the average dwell times of a rail presenting an average flaw in the deterioration states $O k, X_{1}$ and $X_{2}$, as calculated by the physical model.

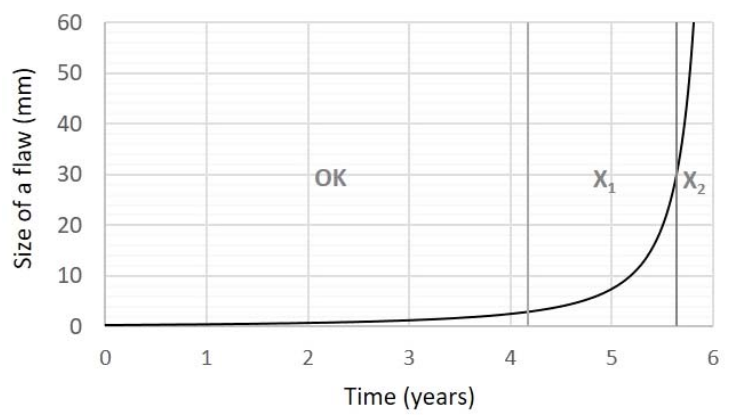

Figure 4: Propagation of a welding defect on a homogeneous track segment of line 7 (central part of the line subjected to dense traffic, tangent, $v=60$ $\mathrm{km} / \mathrm{h}$, rail V52N900).

The sum of the average dwell times in the deterioration states $O K, X_{1}$ and $X_{2}$ corresponds to the average lifetime of a rail presenting a flaw. According to the physical model, this lifetime is equal to 30.8 years on line 7 and to 16.3 years on GPE line 15. This difference can be explained because the axle loads are $25 \%$ heavier on line 15 than on line 7 . Additionally, trains travel at higher speeds on line 15, which further increases the load due to dynamic amplification.

\begin{tabular}{|c|c|c|c|}
\hline \multirow{2}{*}{$\begin{array}{c}\text { From physical } \\
\text { modelling }\end{array}$} & \multicolumn{3}{|c|}{ Average sojourn time (months) } \\
\hline & $\overline{O K}$ & $X_{1}$ & $\mathrm{X}_{2}$ \\
\hline line $7\left(t_{x, L}\right)$ & 310.61 & 52.87 & 6.25 \\
\hline line $15\left(t_{X, L 15}\right)$ & 165.24 & 27.44 & 3,24 \\
\hline $\begin{array}{l}t_{X, L 7} \\
t_{X, L 15}\end{array}$ & 0.53 & 0.52 & 0.52 \\
\hline
\end{tabular}

Table 1: Average dwell times of a flawed rail in the deterioration states considered as calculated by the physical model.

\subsection{Weibull distributions of dwell times}

Table 2 presents the expected value of the Weibull distributions describing the dwell time in Ok, $\mathrm{X}_{1}$ and $\mathrm{X}_{2}$ on lines 7 and 15 and their scale and shape parameters, learnt by maximisation of log-likelihood on the feedback database provided by RATP 
for line 7 and using the methodology introduced in the previous sections for line 15 . Figure 5 gives a representation of these Weibull distributions.

\begin{tabular}{c|c|ccc}
\multicolumn{2}{c|}{ Weibull parameters } & Ok & $\boldsymbol{X}_{1}$ & $\boldsymbol{X}_{2}$ \\
\hline \multirow{3}{*}{ line 7 } & Expected Value & 124.88 & 51.03 & 49.42 \\
\cline { 2 - 5 } & Scale parameter $\alpha$ & 140.26 & 57.62 & 64.96 \\
\cline { 2 - 5 } & Shape parameter $\beta$ & 1.76 & 2.19 & 1.37 \\
\hline \multirow{3}{*}{ line 15 } & Expected Value & 66.43 & 26.49 & 30.68 \\
\cline { 2 - 5 } & Scale parameter $\alpha$ & 74.61 & 29.91 & 33.55 \\
\cline { 2 - 5 } & Shape parameter $\beta$ & 1.76 & 2.19 & 1.37 \\
\hline
\end{tabular}

Table 2: Expected values scale and shape parameters of the Weibull distributions describing the dwell time of a rail in the considered deterioration states.

Intuitively, the dwell time in $X_{2}$ is expected to be shorter than the dwell time in $X_{1}$, which would result in a distribution of $X_{2}$ more to the left than the distribution of $X_{1}$. Surprisingly, this expectation is not the case here. Finally, discussions with the RATP infrastructure managers underline that the available monitoring database deals with a period with mixed quality rails. Indeed, due to a change in rail suppliers, many poor-quality rail sections have been introduced. This point was quickly detected by monitoring services, and sections were replaced. Nevertheless, the activity of this rail supplier induced many events concerning short dwell time in state $X_{1}$ before a replacement for a better-quality rail.

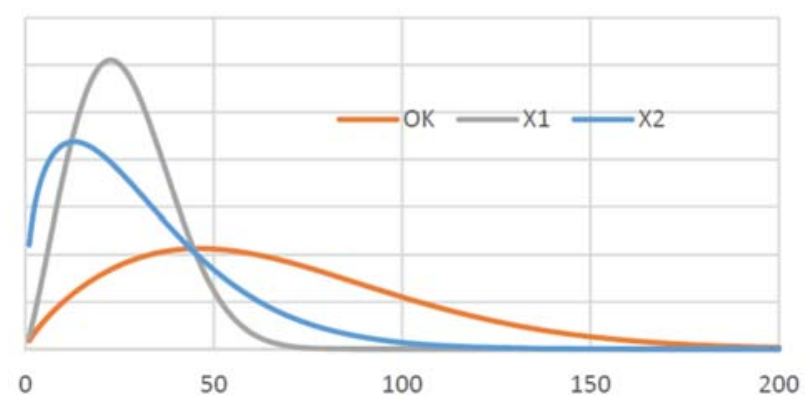

Figure 5: Weibull distributions describing the dwell time (in months) of a rail on line 15 in the deterioration states $O k, X_{1}$ and $X_{2}$.

\subsection{Sensitivity analysis}

As a remainder, a 4 passenger $/ \mathrm{m}^{2}$ load was considered on line 7 during the peak hours and 0.5 during the slack period. In Figure 6, the sensitivity to this assumption in the Weibull rail degradation process on line 15 is assessed.

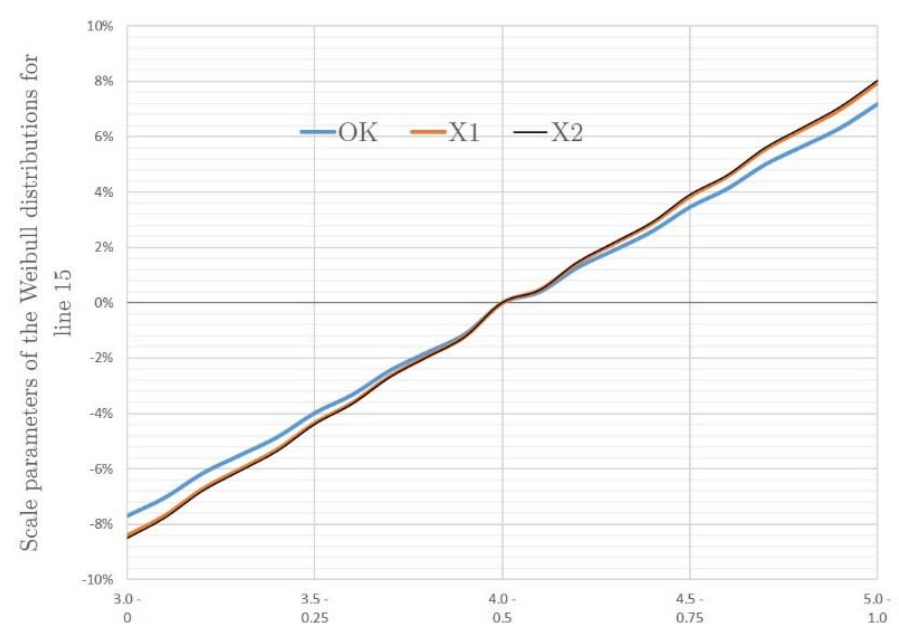

Figure 6: Sensitivity of the Weibull distributions describing the rail deterioration process on line 15 to the assumption concerning the passenger load on line 7 . 
A variation of \pm 1 passenger $/ \mathrm{m}^{2}$ during the peak hours and of \pm 0.5 passenger $/ \mathrm{m}^{2}$ during the slack period induces at most a variation of $8.5 \%$ of the scale parameter of the Weibull distributions. Therefore, we can conclude that the results are relatively robust with regard to the uncertainty of the passenger load on line 7.

\subsection{Some indicator estimation and discussion}

As a conclusion to this section, two experiments will be introduced to underline the kind of learning such an approach can provide. First, the impact of the ultrasonic vehicle (USV) monitoring period on both preventive maintenance actions and the annual number of broken rails will be investigated through Figure 7, introducing the percentage of evolution of these two indicators with respect to the USV period and in comparison with the "standard" scenario considered with a $\mathrm{T}_{0}$ monitoring period. The more frequently tracks are inspected, the earlier preventive actions can be planned and then that fewer broken rails occur is obvious. Nevertheless, with Figure 7, one can evaluate both the necessary "optimal" USV period to decrease the annual number of broken rails by $\mathrm{x} \%$ and the impact on the number of preventive maintenance actions. Conversely, knowing the monitoring demand for the whole network, one can estimate the consequence of the induced monitoring period change on the annual number of broken rails.

Then, during this study, one of the expectations of SGP was both to characterise new monitoring devices according to their needs in terms of infrastructure availability and to determine if a "low detection rate" monitoring device installed on commercial coaches could provide better preventive monitoring of the rails than the USV process.

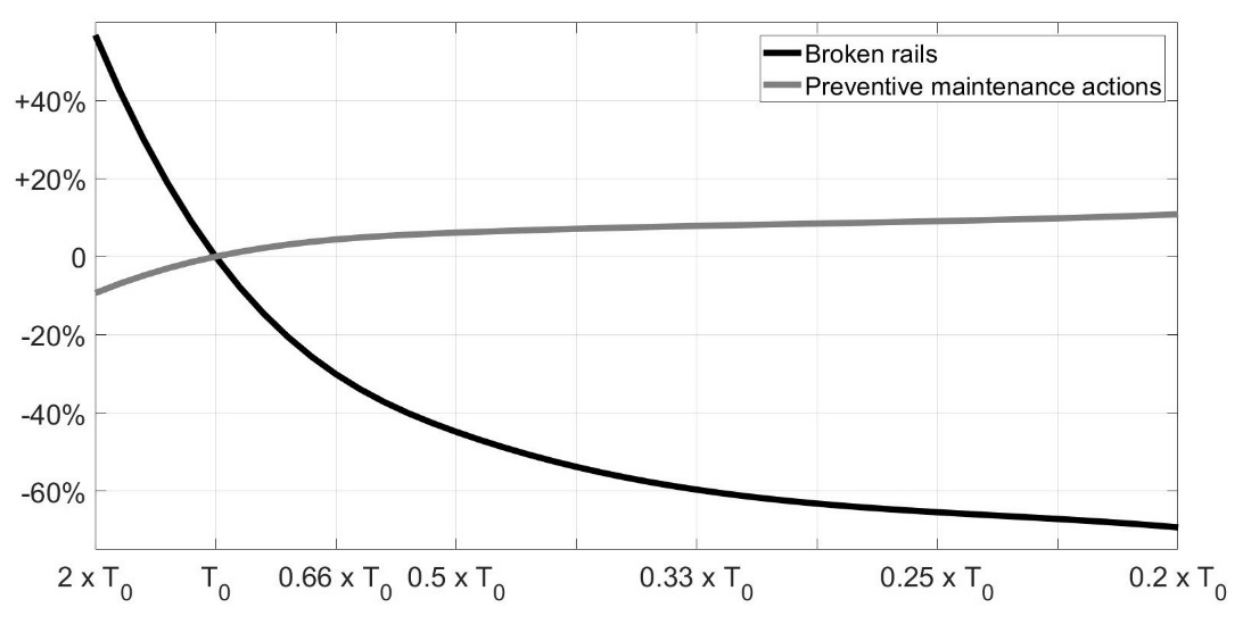

Figure 7: Impact of USV period changes on the annual number of broken rails and preventive maintenance actions. Comparison with a "standard" scenario

Figure 8 introduces the impact of the monitoring period of this "virtual" sensor according to its preventive detection rate on the broken rail index (ratio of annual number of broken rails with the scenario considering the only USV with its "optimal" period estimated through Figure 7). Only scenarios with either the single virtual sensor or the single USV are considered. Scenarios with both sensors were also computed, providing better results than the scenarios introduced in Figure 8, but the aim is here to demonstrate the eventual benefits of onboard "average" monitoring sensors compared with high-level monitoring devices such as the USV. Of course, both approaches will be jointly considered for the final SGP decision.

Finally, we would like to focus on onboard sensors detecting only $5 \%$ of $\mathrm{X}_{2}$ but monitoring infrastructure every day to provide a significant decrease in the annual number of broken rails compared with the standard scenario with only the USV (monitoring rails usually twice a year or every 3 months).

In conclusion, many other results could be introduced in this section, but the aim of this paper is not to detail what SGP did with this decision support tool during the constitution of its safety report but to underline that, even without feedback data, some hybrid approaches considering that the accuracy of a mechanical model with flexible stochastic approaches could be helpful for decision makers to optimise maintenance and monitoring strategies. 


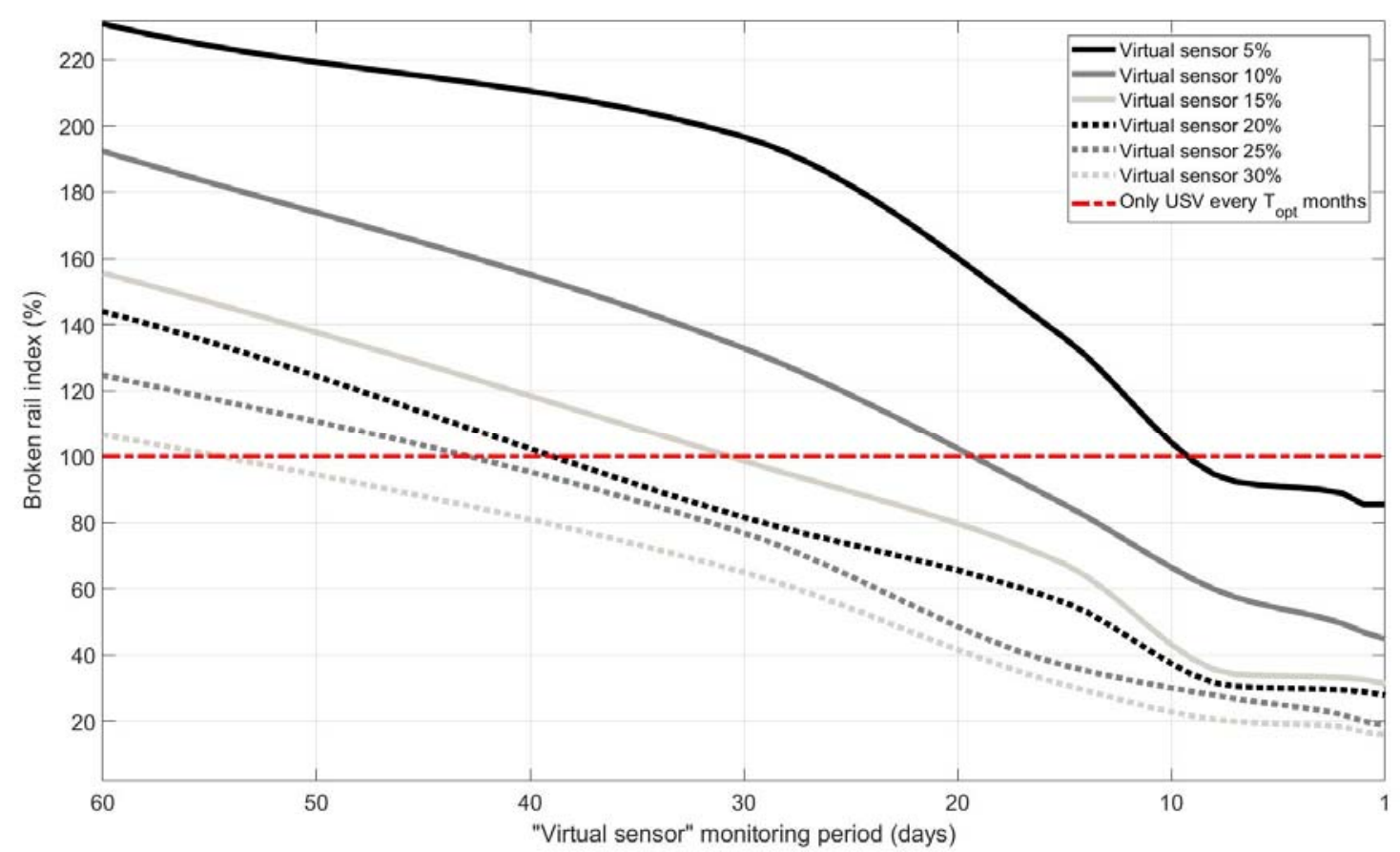

Figure 8: Comparison of broken rail index behaviour for several parameterisations of the "virtual" sensor in comparison with a monitoring strategy limited to the use of a USV.

\section{Conclusions and prospects}

In this paper, an original approach was proposed for the implementation of a decision support tool for evaluating, comparing and optimising several maintenance and monitoring strategies for rails in the context of the upcoming Grand Paris Express network.

Indeed, if reliability analysis tools are generally based on either physical consideration (with analytical models) or, more commonly, stochastic modelling, this second approach needs feedback data for learning parameters, whereas analytical models must be validated by laboratory tests and are therefore applicable for only a very strict set of contextual parameters. This limitation is the reason why previous studies on rail maintenance optimisation, detailed in the literature review, considered stochastic approaches learned on feedback databases provided by infrastructure managers.

In the specific context of the Grand Paris Express, no database was available since such a railway network does not yet exist. To overcome this limitation of the study, an original approach was proposed for combining physical modelling of rail deterioration with reliability statistical approaches. A multibody model of rail degradation was therefore proposed for both a reference Paris metro line and GPE line 15 (considering the specifications of track laying and constitution). Then, adjustment rates were estimated on the mean and variance characterising the time spent in each rail degradation state defined by the UIC standard (No flaw, $X_{1}$ and $X_{2}$ ). These rates were finally used to adjust the dwell time distribution parameters introduced in a Bayesian network-based decision support tool.

Finally, this study enables SGP to evaluate the consequences (in terms of safety, availability, maintainability, etc.) of some of its major decisions such as having a "track circuit free network" as well as to quantify technical recommendations to help infrastructure managers (evaluation of minimal detection threshold for preventive devices, optimal monitoring frequencies, etc.).

The aim of this paper was to introduce a general methodology that accommodates the lack of feedback data for reliability analysis rather than to present results obtained by the SGP during this project. Nevertheless, as an illustration of the opportunities provided by such a decision support tool, several simulations were introduced underlying the ability of the approach to evaluate the optimal ultrasonic monitoring strategy and satisfy the expected availability, safety, and reliability indicators. Finally, considering that, in the model, a "virtual" sensor enables the SGP to determine what should be the features of a new onboard monitoring device, able to provide frequent estimations of the rail state even if its detection abilities are more capable than those of standard ultrasonic vehicles. 
To conclude, the proposed hybrid "mechanico-statistic" approach appears to be a good solution to overcome the lack of feedback data and to evaluate monitoring and maintenance strategies. If the application introduced in this paper was limited to rail maintenance, the proposed approach is, to our mind, perfectly generic and could be applied to several industrial fields.

\section{Acknowledgments}

The authors want to thank the $S G P$ for the trust they placed is us for this sensitive study. We also thank the RATP infrastructure management department for providing extracts of their feedback database, which is required to successfully complete this project.

\section{References}

1. L. Bouillaut, O. François and S. Dubois, "A Bayesian network to evaluate underground rails maintenance strategies in an automation context" Proc IMechE Part O: Journal of Risk and Reliability, vol. 227(4), pp.411-424, 2013, (DOI $10.1177 / 1748006$ X13481306)

2. L. Bouillaut, P. Aknin and R. Donat, "VirMaLab - A generic approach for optimizing maintenance policies of complex systems", $9^{\text {th }}$ World Congress on Railway Research (WCRR), Lille, France, September 2011.

3. K.P. Murphy, "Dynamic Bayesian networks: representation, inference and learning". PhD thesis, University of California, Berkeley, 2002

4. R. Donat, P. Leray, L. Bouillaut, P. Aknin, and S. Bondeux, "A dynamic Bayesian network to represent discrete duration models", Neurocomputing, Vol. 73, pp. 570-577, 2009

5. T. Bendell, "An overview of collection, analysis, and application of reliability data in the process industries", IEEE Transactions on Reliability, Vol. 37(2), pp. 132-137, 1988.

6. L. Bouillaut, R. Donat, A. Neji and P. Aknin, "Estimation of Multi-Components System's reliability: Comparison of two Graphical Model Approaches", 13th IFAC Symposium on Information Control Problems in Manufacturing (INCOM), Moscow, Russia, March 2009.

7. F.V. Jensen, "An introduction to Bayesian networks". UCL Press, 1996.

8. M. Jordan, "Learning in graphical models". Cambridge, MA:MIT Press, 1999.

9. C. Esveld, "Modern Railway Track", MRT Productions, Second Edition, 2001.

10. L.P. Lebet, M. Hirt, "Steel Bridges - Conceptual and Structural Design of Steel and Steel-Concrete Composite Bridges", EPFL Press, Ed. Eyrolles, Paris, 2013.

11. Australian Rail Track Corporation Ltd, "Rails Defects Handbook - Some rail defects, their characteristics causes and control", Engineering practices manual, Civil Engineering, RC 2400, 2006.

12. W. Weibull, "A statistical distribution function of wide applicability", Journal of Applied Mechanics, vol. 18(3), 1958.

13. B. Eickhoff, L. Mazzola, Y. Bezin, G. Tucker, H. Stradtmann, A. Haigermoser, H. Chollet, J. Landais, "Trackloading limits and cross acceptance of vehicle approvals". Proc IMechE Part F: Journal of Rail and Rapid Transit, vol. 229(6), 2015.

14. T. H. Lin and Y. M. Ito, "Fatigue crack nucleation in metals", Proceedings of the National Academy of Sciences, vol. 62, 1969.

15. V. A. Profillidis, "Railway engineering", Second edition, pp. 94-95, Ashgate publishing, Aldershot UK, 2000.

16. Y. Zhu, "Adhesion in the wheel-rail contact", Doctoral thesis, Royal Institute of Technology of Stockholm, ISBN 978-91-7501896-6.

17. S. Takahashi, T. Sasaki, Y. Kondoh and Y. Hirose, "Residual stress evaluation of railway rails", JCPDS - International centre for diffraction data, ISSN 1097-002, pp. 240-247, 2009.

18. Z. Cai, M. Nawafune, N. Ma, Y. Qu, B. Cao and H. Murakawa, "Residual stresses in flash butt welded rail", Transactions of Joining and Welding Research Institute, vol. 40(1), 2011.

19. R. Ferrara, "A numerical model to predict train induced vibrations and dynamic overloads", Doctoral thesis, University Montpellier II - Sciences et Techniques du Languedoc, 2013.

20. S. Kumar, Study on rail breaks: Associated risks and maintenance strategies", Technical report, Lulea University of Technology, Sweden, 2006. 